\title{
Partially Estimated Polynomial MEMS Sensor Calibration
}

\author{
Andreas Dickow, Gregor Feiertag \\ Laboratory for electronic Packaging, Munich University of Applied Sciences, Lothstrasse 64, D-80335 Munich \\ dickow@hm.edu,gregor.feiertag@hm.edu
}

\begin{abstract}
MEMS sensors are individually calibrated to reach a high sensing accuracy. For calibration certain measurement points are selected as reference and used to calculate the parameters of a calibration polynomial function. To minimize the calibration costs, MEMS sensor calibration should be performed using as few calibration points as possible. We propose a calibration approach which gives good accuracy with less measurement points. The approach combines a systematic calibration method with statistical parameter estimation, to find appropriate measurement points for calibration and minimize the number of calibration points. An experiment with a set of commercial barometric MEMS pressure sensors validates the proposed method for MEMS barometric pressure sensors.
\end{abstract}

Key words: sensor calibration, calibration polynomial, MEMS sensors, parameter estimation, analytic solution.

\section{Introduction}

MEMS sensors are used for many different sensing applications. For some applications, it is necessary to compensate the cross sensitivities e. g. to temperature. Sensor calibration techniques are used to compensate cross sensitivities and nonlinearities [1-5]. An overview of sensor calibration techniques is given by [6]. In [6] model based and model free calibration methods are distinguished. Model free methods use a cost function and a optimization method like the Nelder-Mead simplex algorithm.

Model based calibration methods can include cross-sensitivity, sensor nonlinearity and noise suppression in a model based on measurements or physical considerations. It relies on polynomials or differential equations, fitting measurement results to a given measurement reference.

In this paper the subject of investigation is model based calibration using polynomials. This is a widely used method in calibration of low cost MEMS sensors [3,4]. The calibration approach presented here combines a systematic methodology for finding calibration points, presented in [7] and [8], with process statistics to minimize measurement points needed for calibration and at the same time maximize the calibration quality for a calibration polynomial choosen.

\section{Polynomial calibration}

Polynomial calibration linearizes sensor output using a linear process model

$y_{\text {ref }}=X \beta+\varepsilon$,

where $\mathbf{y}_{\text {ref }}$ is a vector of sensor reference values measured, $\mathbf{X}$ is a matrix of functions of sensor raw values, $\boldsymbol{\beta}$ is a vector of calibration parameters and $\boldsymbol{\varepsilon}$ is a calibration error term. Choosing a simple second order polynomial calibration with only one parameter leads to a calibration model

$y=\beta_{0}+\beta_{1} x+\beta_{2} x^{2}$.

The model error

$$
\begin{aligned}
& \varepsilon_{i}=\left|y_{\text {ref }, i}-y_{i}\right|=\left|y_{\text {ref }, i}-\beta_{0}-\beta_{1} x_{i}-\beta_{2} x_{i}^{2}\right|, \\
& \forall i \in(1, N)
\end{aligned}
$$

is minimized by using least squares on the quadratic norm of $\varepsilon \in \mathbb{R}^{N}$ for all $N$ measurement points for calibration, where $y_{\text {ref }}$ is the reference value for calibration. To determine a unique analytic solution for Eq. (3), $\mathrm{N}=3$ independent measurement points are needed.

\section{Choosing measurement points}

The following scenario considers that there are $\mathrm{M}$ measurement points available for sensor calibration and $\mathrm{M}>\mathrm{N}$. As only $\mathrm{N}$ points are 
needed to calibrate a sensor with a polynomial of order $\mathrm{O}=\mathrm{N}-1$. One can now choose, which measurement point tuple $t$ out of $\mathrm{K}=\left(\begin{array}{l}M \\ N\end{array}\right)$ possible options is taken for sensor calibration.

In [7] and [8] we suggested to calculate the calibration error for all $\mathrm{K}$ calibration options and then select

$$
t^{*} \rightarrow \min \left(\varepsilon_{k}\right) \forall k \in K .
$$

This systematic calibration point selection method is limited by the amount of entries in $\mathrm{K}$. Having more than one independent variable to test against (temperature, strain, pressure, humidity etc.), with several conditions for each variable, the task of calculating all possible measurement point variations can lead to computational problems, as the computation time is a limiting factor for this approach.

\section{Calibration procedure}

For a given set of calibration reference measurement points $M$ and a one dimensional polynomial

$$
y=\beta_{0}+\beta_{1} x+\cdots+\beta_{N-1} x^{N-1},
$$

with $\mathrm{N}<\mathrm{M}$, there are $\mathrm{K}=\left(\begin{array}{l}M \\ N\end{array}\right)$ possibilities to determine a analytic solution for the parameter fitting problem, if the system is not overdetermined. For every $\mathrm{k} \in \mathrm{K}$ the analytic solution is calculated (Least Square algorithm, when more measurement points than necessary should be considered), leading to parameter vectors $\left(\boldsymbol{\beta}_{1}, \boldsymbol{\beta}_{2}, \ldots, \boldsymbol{\beta}_{\mathrm{K}}\right) \in \mathbb{R}^{\mathrm{N}}$. In a multi-dimensional case with each dimension having the same order

$y=\sum_{j=1}^{R} \sum_{i=0}^{N-1} \beta_{i, j} x_{j}^{i}$,

where $\mathrm{R}$ denotes the number of independent parameters used for calibration.

For each vector $\boldsymbol{\beta}_{\mathrm{k}}, \mathrm{k} \in(1, \ldots, \mathrm{K})$, the root mean squared calibration error

$$
\varepsilon_{M}=\sqrt{\frac{1}{M-1} \sum_{i=1}^{M} \varepsilon_{i}^{2}}
$$

is used to compare each set of parameters. The error results with the highest $99 \%$ of all error results are dismissed. The remaining $1 \%$ of best calibration error parameter sets are investigated further.

\section{Example}

A batch of 1000 sensors is calibrated with two out of eight available measurement points. The calibration polynomial $\mathrm{y}^{\boldsymbol{*}}=\boldsymbol{\beta}_{0}+\boldsymbol{\beta}_{1} x_{1}$ is used.

To find a unique calibration solution, at least two measurement points are necessary to calculate $\boldsymbol{\beta}$. The polynomial parameters are identified for each possible measurement point combination $t_{i}=\left(\mathrm{t}_{1}, \mathrm{t}_{2}\right), \quad \mathrm{i} \in(1, \ldots, \mathrm{N})$, where $N=\left(\begin{array}{l}8 \\ 2\end{array}\right)=28$ possible combinations of measurement point values $t_{1}$ and $t_{2}$ exist. After calculating the calibration error using Eq. (7) for each calibration option, the worst $99 \%$ of calibration tuples $t$ are discarded. For a batch of 1000 sensors, 280 calibration point tuples remain. The results can be interpreted in a distribution plot, as Fig. (1) shows. The value of the measurement point is plotted on the $\mathrm{x}$-axis. In the upper plot, the first measurement point used for calibration $t_{1}$ is shown. In the lower plot, the second point $t_{2}$ is depicted. Taken from Fig. (1), the probably best selection of calibration points would be $t^{*}=(2,4)$.

A restriction

$$
t \rightarrow\left(t_{i}, t_{j}\right), t_{i}<t_{j} \forall t_{i}, t_{j} \in \mathbb{R}
$$

Is added to separete the calibration point distributions in Fig. (1). In general

$$
t \rightarrow\left(t_{1}, \ldots, t_{N}\right), t_{1}<t_{2}<\ldots<t_{N} \forall t_{1}, \ldots, t_{N} \in \mathbb{R}
$$

is applied to the proposed calibration method.
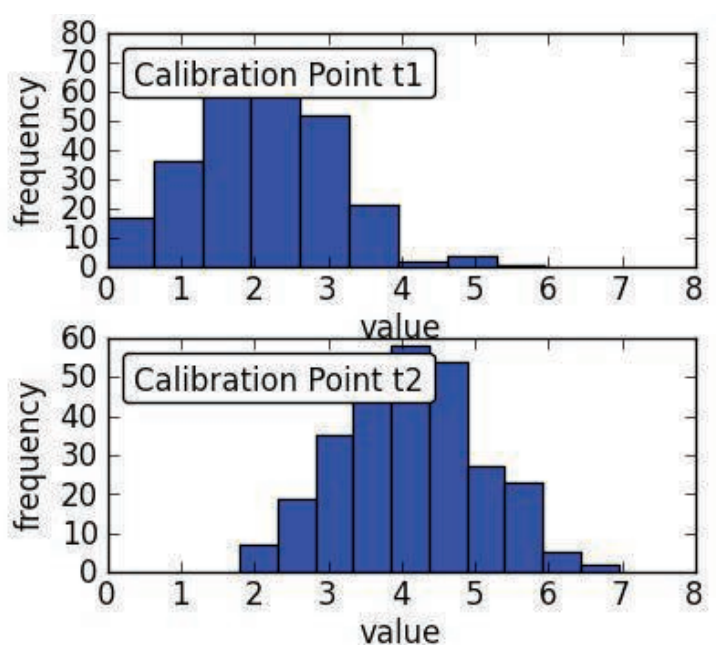

Fig 1. Distribution of best $1 \%$ calibration points for a batch of 1000 sensors calibrated with two parameters and tested against 8 measurement points.

\section{Statistical offset correction}

In industrial scale MEMS sensor production, saving calibration points leads to significant 
time savings. Therefore, the following design objective should be fulfilled:

Calibrate sensors with maximum linearity under the restriction of using as few calibration points as possible.

To do so, the proposed systematic calibration procedure is enhanced by adding a function of static offset values taken from measurement statistics. An improvement of sensor readout can be achieved, if the order of the statistical correction polynomial is higher than the order of the calibration polynomial used. Therefore the correction polynomial mainly enhances a low order calibration polynomial by adding higher order polynomial terms, taken from statistics. The statistical correction procedure is described as follows:

From production, a control sample consisting of calibrated sensors is taken to determine the statistical correction function. Within the control sample, the sensor's nonlinearity is investigated in measurements at defined conditions. After measurement values were recorded, the mean value of the sensor's readout values is calculated. The error between the polynomial fitting function of the mean sensor readout and the reference vaules is minimized, by using least squares approximation over all available mean measurement values. After the polynomial approximation is calculated, the function identified is subtracted from each readout calibrated. This procedure is shown in Fig. (2). The mean readout (green line) is used to linearize the sensor readout values (red lines). By subtracting the identified function from the sensor readout, the calibrated sensors readout is linearized against a well calibrated reference sensor. The linearization result is depicted as blue lines.

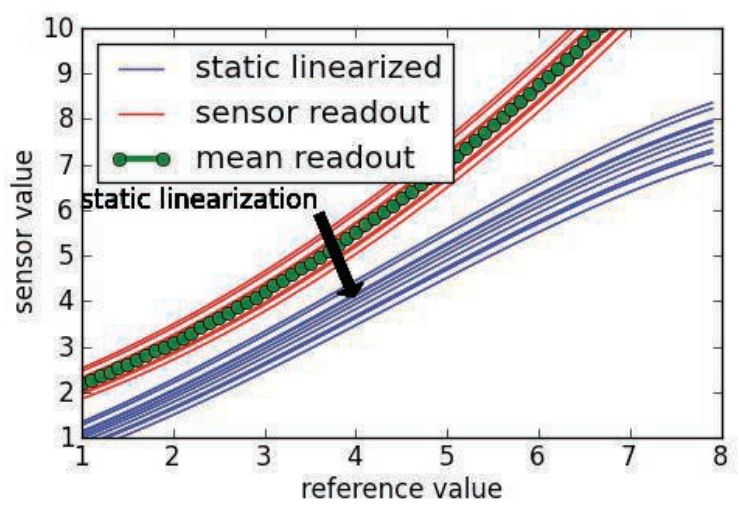

Fig 2. Linearization of linear calibrated sensor readout using a statistical determined correction polynomial of second order.

Algorithm
Step1: define a correction polynomial

$f_{\text {static }}^{*}\left(y_{j}\right)=\sum_{j=1}^{R} \sum_{i=0}^{Q-1} \alpha_{i, j} y_{j}^{i}, Q>N$,

where $Q$ denotes the highest order used for calibration, $\boldsymbol{\alpha}$ is a matrix of parameters describing the static correction function $f^{*}$ static and $y_{j}$ is the mean sensor readout value with $\mathrm{j}$ $\in(1, \ldots, R)$.

Step 2: The unknown parameters a are calculated by solving

$\min \left(\varepsilon_{k, \text { static }}\right) \forall k \in K$, where

$\varepsilon_{k, \text { static }}=\left|y_{\text {ref }}-f_{\text {static }}^{*}\right|$,

Where $y_{\text {ref }}$ is a vector of reference values of a well calibrated reference sensor.

Step 3:

After the correction function $f^{*}$ static is calculated, it can be used to correct the calibrated sensor output with

$y_{\text {corr }}=y_{\text {poly }}^{*}-f_{\text {static }}^{*}\left(y_{\text {poly }}^{*}\right)$.

\section{Application example - MEMS barometers}

In this section an application example is presented for the proposed combination of systematic calibration and statistical parameter correction. MEMS barometers are choosen as an example because they have a strong crosssensitivity to temperature and nonlinear behavior over pressure and temperature.

\section{Setup}

The experiment investigates the influence of statistical compensation on the avarage pressure sensing error for 38 MEMS barometers of the type EPCOS T5400. The sensors are calibrated for operation at a temperature range from $-40{ }^{\circ} \mathrm{C}$ to $90{ }^{\circ} \mathrm{C}$ and barometric pressures from $300 \mathrm{hPa}$ to 1100 $\mathrm{hPa}$.

Measurement data is discretized in $10{ }^{\circ} \mathrm{C}$ steps and $100 \mathrm{hPa}$ steps, resulting in a grid of 126 measurement points were recorded. A General Electric PACE 6000 pressure controller is used to control the barometric pressure applied to the sensors and record the pressure reference value $y_{\text {ref }}$. Temperature is controlled by Peltier-elements and type $\mathrm{K}$ thermocouples attached within the pressurized test volume. Measurement data is recorded in ascending order from $-40^{\circ} \mathrm{C}$ to $90^{\circ} \mathrm{C}$ and $300 \mathrm{hPa}$ to 1100 $\mathrm{hPa}$ at each temperature step. 


\section{Systematic calibration}

For the example a two dimensional first order calibration polynomial

$y_{\text {poly }}^{*}=\beta_{0}+\beta_{1} x_{P}+\beta_{2} x_{T}+\beta_{3} x_{T} x_{P}$

is choosen, where $x_{T}$ is the barometers uncalibrated temperature readout and $x_{P}$ is the barometers uncalibrated pressure readout. To determine the parameters of Eq. (13) at minimum 4 measurement points are necessary. The measurement points are selected using the systematic calibration method, described in the previous sections. For each sensor investigated, the probably best calibration option is taken from the distribution plot shown in Fig. (3). In Fig. (3) a measurement tuple consists of $\left(\left(T_{1}, P_{1}\right),\left(T_{2}, P_{2}\right),\left(T_{3}, P_{3}\right),\left(T_{4}, P_{4}\right)\right)$, where $\mathrm{T}\left({ }^{\circ} \mathrm{C}\right)$ is a temperature measurement point and $\mathrm{P}(\mathrm{hPa})$ is a pressure measurement point. The most common measurement point combination $t^{*}=\left[\left(-10^{\circ} \mathrm{C}, 300 \mathrm{hPa}\right),\left(80^{\circ} \mathrm{C}, 300\right.\right.$ $\left.\mathrm{hPa}),\left(-20^{\circ} \mathrm{C}, 1100 \mathrm{hPa}\right),\left(70{ }^{\circ} \mathrm{C}, 1100 \mathrm{hPa}\right)\right]$ is used in the following to calibrate the MEMS barometers.

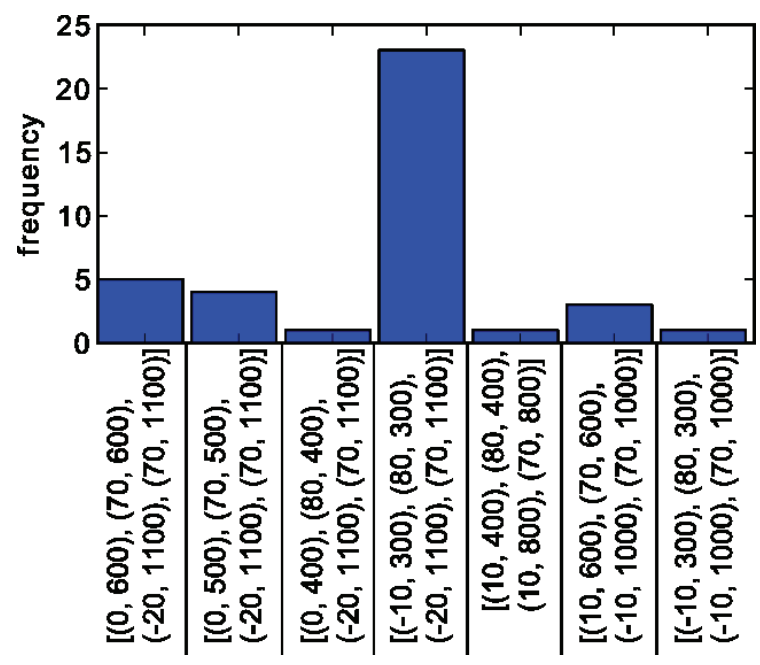

Fig 3. Frequency of best calibration options for 38 MEMS barometers calibrated with a first order polynomial $(x$ values are tuples $\left(\left(T_{1}, P_{1}\right),\left(T_{2}, P_{2}\right),\left(T_{3}, P_{3}\right),\left(T_{4}, P_{4}\right)\right)$, where $T$ denotes temperature in ${ }^{\circ} \mathrm{C}$ and $P$ is barometric pressure in $h P a)$.

\section{Statistical linearization}

After all barometers are calibrated using $t^{*}$, they are tested against all 126 measurement points. As described in the section before, a statistical correction function is calculated using the least square approximation from Eq. (11). A polynomial of order two in pressure and in temperature is used as statistical correction function.

A comparison between the uncompensated and the statistical compensated sensor calibration is made. In addition, another comparison is made between the statistical compensated sensors, calibrated with a four parameter first order polynomial + statistical compensation, and nine parameter second order in temperature and pressure polynomial calibrated sensors. This second comparison is intended to show, how much worse the statistical estimation of a second order polynomial calibration is, compared to a second order polynomial calibration with measured values.

The comparison is depicted in Fig. (4). In the upper plot the first order polynomial calibration is compared to the statistical compensated calibration. Both calibrations require 4 measurement points, if statistics are available. In the lower plot a second order polynomial calibration is compared to the first order polynomial calibration with statistical compensation. Here the second order calibration requires 9 measurement points, compared to 4 measurement points for the statistical compensated calibration. In both plots the measurement temperature is used as $\mathrm{x}$-axis and the mean error over pressure range is used as y-axis. Uncompensated calibration is marked gray and compensated calibration is marked red. The vertical lines depict the spread of the mean error, as 38 sensors were investigated.

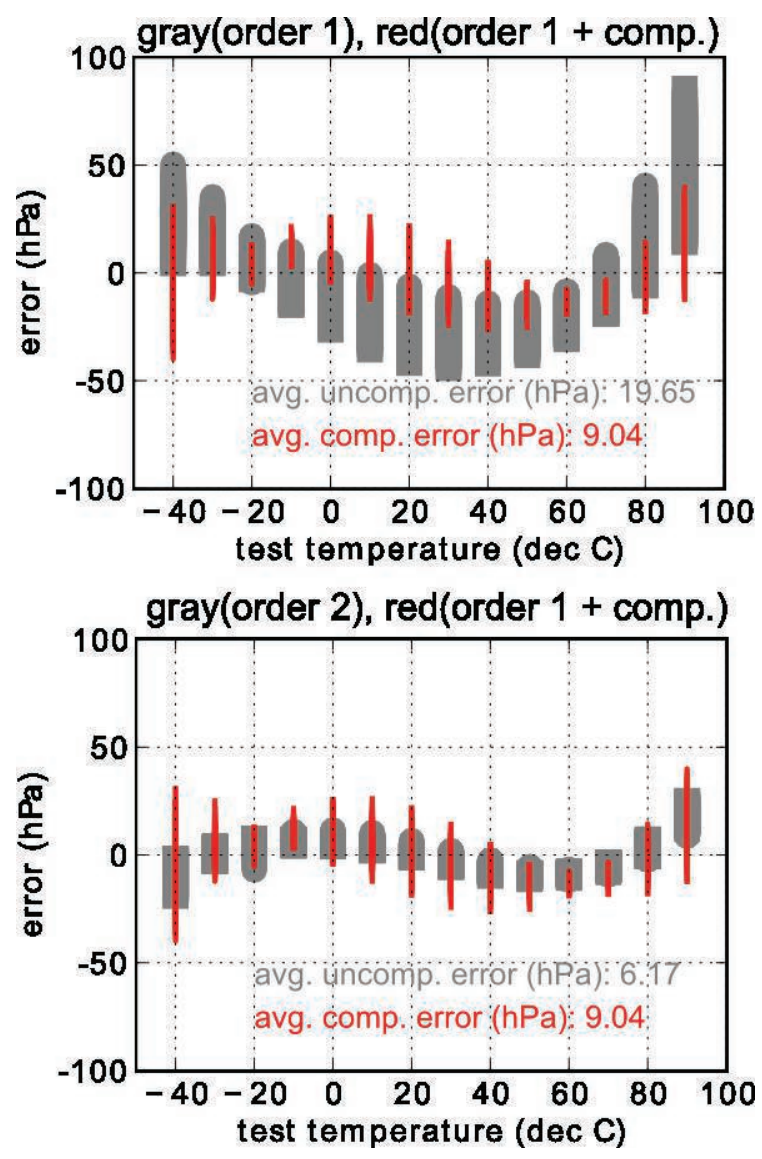

Fig 4. Statistical linearized sensors (red) show less measurement error than first order calibrated sensors 
without compensation (upper plot, in gray), and slightly more measurement error than second order uncompensated sensors (lower plot, in gray).

As shown in Fig (4), the statistical compensation results in an average pressure error of $9.04 \mathrm{hPa}$ over full pressure and temperature range (avg-spec-error) specified. Compared to the initial avg-spec-error 19.65 $\mathrm{hPa}$ of a systematically calibrated barometer, this is a improvement of more than a factor two.

Comparing the avg-spec-error of the compensated first order calibrated sensor with the avg-spec-error $6.17 \mathrm{hPa}$ of the second order calibrated barometer, the compensated sensor performs more than $50 \%$ worse. But on the other hand, less than half of the second order calibration's measurement points are required to calibrate the statistical compensated MEMS barometers.

So for the barometer example, the design objective of calibrating with maximum linearity using as less as possible measurement points for calibration is met by using a combination of systematic calibration and statistical parameter estimation, with a little trade-off in sensor linearity.

\section{Concluding remarks and outlook}

For a MEMS barometer example, it is shown that sensors can be calibrated well with a systematically determined calibration polynomial in combination with a statistical nonlinearity compensation. A method for statistical estimation of a calibration offset function is introduced. The statistic compensation method is applied to sensors, which were calibrated with polynomials using as few measurement points as possible. The probably best measurement point combinations for calibration were determined with a systematic approach.

The method is limited to low order polynomials for computational complexity issues. It is intended to apply the method to other types of MEMS sensors like humidity sensors and gyroscopes.

\section{Acknowledgements}

This work was supported by the Bavarian ministry of economic affairs within the researchproject MEMSBaro.

\section{References}

[1] S.B. Crary, W.G. Baer, J.C. Cowles and K.D. Wise, Digital compensation of high-performance silicon pressure transducers, Sensors and Actuators A 2I-23, 70-72 (1990); doi:10.1016/0924-4247(90)85014-U
[2] K. Lyahou, G. Van der Horn, J. Huijsing, A NonIterative, Polynomial, 2-dimensional calibration method implemented in a microcontroller, IEEE Instrumentation and Measurement Technology Conference June 4-6, 1996, Brussels, Belgium (1996); doi: 10.1109/IMTC.1996.507349

[3] W.T. Bolk, A general digital linearising method for transducers, J. Phys. E: Sci. Instrum. 18, 61 - 64 (1985); doi:10.1088/0022-3735/18/1/016

[4] T-R. Hsu, MEMS \& Microsystems: Design, Manufacture, and Nanoscale Engineering, 2.Ed. Wiley \& Sons, Hoboken, New Jersey (2008);

[5] Y. Günhan,D. Ünsal, Polynomial Degree Determination for Temperature Dependent Error Compensation of Inertial Sensors, Position, Location and Navigation Symposium - PLANS 2014, 2014 IEEE/ION, 5-8 May 2014, Montery Candada, 1209-1212 (2014); doi:10.1109/PLANS.2014.6851494

[6] J. E. Brignell, Digital compensation of sensors, J. Phys. E: Sci. Instrum. 20 , 1097-1102 (1987); doi:10.1088/0022-3735/20/9/005

[7] A. Dickow, G. Feiertag, A framework for Calibration of barometric MEMS pressure sensors, Procedia Engineering: Eurosensors Conference 2014. 87, 1350-1353 (2014); doi:10.1016/j.proeng.2014.11.716

[8] A. Dickow, G. Feiertag, A systematic MEMS calibration framework, (accepted at) Journal of sensors and sensor systems (2015). 